concern, particularly around diabetes and heart disease. ${ }^{1}$

In light of this renewed drive towards preventative healthcare in the UK, combined with the burden of periodontal disease on our ageing population, treatments that allow for proactive patient self-management of this condition should be welcomed by patients and dentists alike. It is imperative that I continue to strive to educate patients on the signs and symptoms of periodontal disease and ensure that they are well equipped with the necessary selfmanagement techniques for effective preventative homecare.

H. Mostafa By email

1. Mostafa H. Survey of 66 periodontal specialists and general dental practitioners conducted in September 2011.

DOI: 10.1038/sj.bdj.2011.1011

\section{AN ADVISED CHOICE}

Sir, many GDPs look at some point in their career to the possibility of studying for a postgraduate qualification. This journal is full of adverts trying to catch the eye of the dentist looking to enhance their skills, develop their practice or reinvigorate their career. This was a path I took when I committed to a part time MSc at the University of Edinburgh. I looked to this opportunity to learn new skills and provide a stimulating challenge. Unfortunately the course failed to live up to my understandably high expectations. I left after two years, comfortably passing the diploma, but deciding against taking the qualification further due to my dissatisfaction. The course floundered under a weight of poor planning and organisation, with fundamentals, such as the need to provide feedback, being ignored.

I write this letter not to discourage my colleagues from this path, but to encourage them to enter into postgraduate education with their eyes open. The University of Edinburgh is a very highly regarded institution with an international reputation; however, the nature of the rewarding body is clearly no guarantee of course quality. I would advise my fellow dentists to do a substantial amount of research before committing to a course, question- ing levels of support that they should expect and analysing the timetabling of any coursework they will be required to complete. In my experience it is easy to describe a course as, 'PT and modular, being ideally suited to the busy GDP', while failing to recognise even the most obvious commitment, such as being sympathetic to a parent's need to take holidays at fixed times.

I would like to think that my problems were very much the minority; however, I hope my words will allow anybody interested in this career opportunity to make a more advised choice. Further details can be found in my course review on the Hotcourses. com website.

S. Steven, Edinburgh DOI: 10.1038/sj.bdj.2011.1012

\section{DISEASE RISK}

Sir, recently there has been a paradigm shift in our understanding of periodontal disease. The current consensus is on a risk based approach in evaluating patients for periodontal disease and subsequently for its treatment.

The American Academy of Periodontology has devised a simple tool for the general public to self assess their periodontal risk. It can be accessed by the general public on their website at http:// www.perio.org/consumer/4a.html

It contains a set of 12 questions and once answers are submitted, a proprietary calculation determines the individual's risk for periodontal disease. It categorises the risk into low, medium or high and generates a report. This Periodontal Disease Risk Score and the information contained in the report is intended to help educate patients about common risk factors related to periodontal diseases and to assist in the decision of when referral to a periodontist would be advisable.

It is surprising that the British Society of Periodontology does not have a periodontal risk assessment tool for the general population and their website refers patients to the American Academy of Periodontology website (http:// www.bsperio.org.uk/patients/) for extensive information.

Z. Imran, Dundee DOI: 10.1038/sj.bdj.2011.1013 